\title{
Broad neutralization of Influenza virus and implications for universal therapies
}

\author{
Ian Andrew Wilson $^{1}$ \\ ${ }^{1}$ The Scripps Research Inst., La Jolla, United States \\ E-mail: wilson@scripps.edu
}

Until relatively recently, most antibodies to influenza virus were thought to be strain-specific and protect only against highly related strains within the same subtype. Since 2008, many human antibodies have been isolated that neutralize across different subtypes and types of influenza viruses through binding to functionally conserved sites. The major surface antigen, the hemagglutinin (HA), of influenza virus is the main target of these neutralizing antibodies. Crystal structures of several broadly neutralizing human antibodies (bnAbs) with different HA strains, subtypes and types have identified their binding epitopes as the highly conserved functional sites on the HA fusion domain (stem) in influenza A and B viruses, as well as the receptor binding site (reviewed (1)). Characterization of these broadly neutralizing antibodies along with their mode of binding and neutralization has provided exciting new opportunities for structure-assisted vaccine and therapeutic design to afford greater protection against influenza viruses $(1,2)$. Indeed, mini-HA immunogens designed to mimic the highly conserved HA stem elicited protective responses against different influenza subtypes, such as H1N1 and H5N1, in mice and monkeys (2), thereby illustrating promising proof of concept for development of a more universal flu vaccine. We also recently determined the structure of a broad anti-viral small molecule arbidol to elucidate its binding site and mechanism of action (3). This work has been supported by NIH grants AI117675, AI117905, HHSN2722014, GM094586 and Janssen/Crucell.

Lee, P.S. and Wilson, I.A. (2015). Curr Top Microbiol Immunol 386:323-41.

Impagliazzo et al. (2015). Science 349:1301-1306.

Kadam, R. and Wilson, I.A. (2017). PNAS 114:206-214.

Keywords: influenza virus, antibodies, vaccine 